\title{
Integration of Atomistic Simulation with Experiment Using Time-Temperature Superposition for a Cross-Linked Epoxy Network
}

Ketan Khare, Frederick R. Phelan Jr.

Submitted date: 26/06/2019 Posted date: 27/06/2019

Licence: CC BY-NC-ND 4.0

Citation information: Khare, Ketan; Phelan Jr., Frederick R. (2019): Integration of Atomistic Simulation with Experiment Using Time-Temperature Superposition for a Cross-Linked Epoxy Network. ChemRxiv. Preprint.

Quantitative comparison of atomistic simulations with experiment for glass-forming materials is made difficult by the vast mismatch between computationally and experimentally accessible timescales. Recently, we presented results for an epoxy network showing that the computation of specific volume vs. temperature as a function of cooling rate in conjunction with the time-temperature superposition principle (TTSP) enables direct quantitative comparison of simulation with experiment. Here, we follow-up and present results for the translational dynamics of the same material over a temperature range from the rubbery to the glassy state. Using TTSP, we obtain results for translational dynamics out to $10^{9} \mathrm{~s}$ in TTSP reduced time - a macroscopic timescale. Further, we show that the mean squared displacement (MSD) trends of the network atoms can be collapsed onto a master curve at a reference temperature. The computational master curve is compared with the experimental master curve of the creep compliance for the same network using literature data. We find that the temporal features of the two data sets can be quantitatively compared providing an integrated view relating molecular level dynamics to the macroscopic thermophysical measurement. The time-shift factors needed for the superposition also show excellent agreement with experiment further establishing the veracity of the approach.

File list (3)

EpoxyMSD_Main.pdf (848.51 KiB)

view on ChemRxiv - download file

EpoxyMSD_SP.pdf (1.16 MiB)

view on ChemRxiv - download file

EpoxyMSD_Video.mp4 (17.26 MiB)

view on ChemRxiv • download file 


\title{
Integration of Atomistic Simulation with Experiment using Time-Temperature Superposition for a Cross-linked Epoxy Network
}

\author{
Ketan S. Khare*,t,‡ and Frederick R. Phelan, Jr.*, \\ †Department of Physics, Georgetown University, Washington, DC 20057, United States \\ ‡Material Science and Engineering Division, National Institute of Standards and Technology, Gaithersburg, MD 20899, United States
}

\begin{abstract}
Quantitative comparison of atomistic simulations with experiment for glass-forming materials is made difficult by the vast mismatch between computationally and experimentally accessible timescales. Recently, we presented results for an epoxy network showing that the computation of specific volume vs. temperature as a function of cooling rate in conjunction with the time-temperature superposition principle (TTSP) enables direct quantitative comparison of simulation with experiment. Here, we follow-up and present results for the translational dynamics of the same material over a temperature range from the rubbery to the glassy state. Using TTSP, we obtain results for translational dynamics out to $10^{9} \mathrm{~s}$ in TTSP re-
\end{abstract}

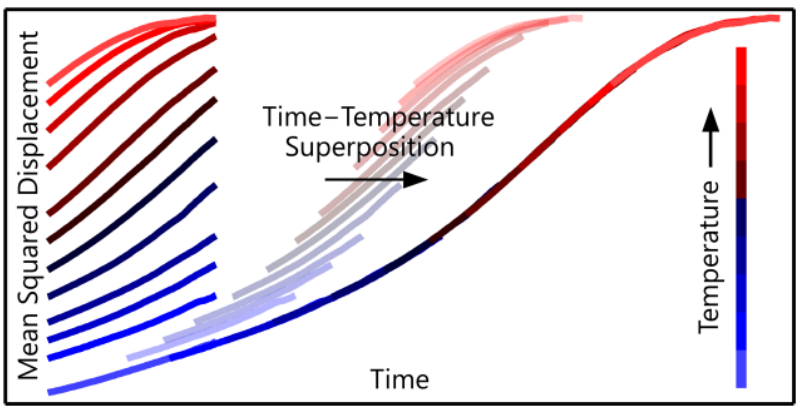

duced time - a macroscopic timescale. Further, we show that the mean squared displacement (MSD) trends of the network atoms can be collapsed onto a master curve at a reference temperature. The computational master curve is compared with the experime ntal master curve of the creep compliance for the same network using literature data. We find that the temporal features of the two data sets can be quantitatively compared providing an integrated view relating molecular level dynamics to the macroscopic thermophysical measurement. The time-shift factors needed for the superposition also show excellent agreement with experiment further establishing the veracity of the approach.

The extreme mismatch between the timescales that are accessible via atomistic simulations compared to experiment is a major barrier to the integration ${ }^{1}$ of the two methods for materials research. For viscoelastic materials, rate effects are an inalienable aspect of their thermomechanics. Thus, such effects are of direct relevance to their applications. Recently, we have shown ${ }^{2}$ that the study of the temperature trend of volumetric behavior as a function of the cooling rate can be used to compare experiment with atomistic simulations for crosslinked epoxy networks despite the ten order magnitude mismatch in the respective rates. Further, we used the time-temperature superposition principle (TTSP) to successfully compare the rate-dependence of computational $T_{\mathrm{g}}$ 's with experimental literature data. ${ }^{2}$ To our knowledge, this was the first quantitative comparison of volumetric trends for thermosets. ${ }^{3}$

TTSP has also been previously invoked by one of us to collapse the temperature trends of Young's modulus of atomistic models of crosslinked epoxy studied at different strain rates. ${ }^{4}$ The resulting master curve showed reasonable agreement with the experimental master curve of storage modulus. ${ }^{4}$ However, Young's modulus and other quantities that rely on the virial stress tensor are subject to large statistical fluctuations, and hence, a relatively high degree of uncertainty. ${ }^{3}$ Our focus here is the study of the temperature trend of the mean squared displacement (MSD), which can be readily and accurately calculated from atomistic simulations.

In the literature, reports of MSD trends of cross-linked epoxy using atomistic simulations that extend to nanosecond timescales are rare. ${ }^{5-}$ ${ }^{7}$ Lin and Khare ${ }^{5}$ provided a detailed analysis of the dynamics of a crosslinked epoxy network using atomistic simulations by calculating the MSD trends of two sets of atoms as a function of temperature across $T_{\mathrm{g}}$ It was shown for both sets that: (1) the MSD trends with time $(t)$ have two subdiffusive regions that transition from roughly $t^{0.2}$ to $t^{0.5}$ dependence; and, (2) the expected plateau in the MSD trends at long times due to topological localization was not found.
Here, we study the translation dynamics of the same epoxy network whose volumetric behavior was recently characterized by us. ${ }^{2}$ This network is formed by the polycondensation of Epon $1001 \mathrm{~F}^{8,9}$ and 4,4'-diaminodiphenyl sulfone $\left(4,4^{\prime}\right.$-DDS). The choice of the network chemistry (Figure 1) was in part motivated by the availability of experimental data. ${ }^{10-13}$ Simulations were started using snapshots for each of five replicas from the previous work. ${ }^{2}$ We selected the snapshots from the slowest cooling rate $\left(5.556 \times 10^{9} \mathrm{~K} \mathrm{~s}^{-1}\right)$ temperature series. All simulations were performed using the LAMMPS simulation package. ${ }^{14}$ The molecular models were described using the all-atom general AMBER force field (gAff) ${ }^{15-17}$ and the partial charges were calculated using Austin Model 1 with bond charge correction (AM1-BCC) method. ${ }^{18-20}$ Further simulation details are available in our recent work. ${ }^{2}$

"Error bars" in the figures or uncertainty associated with quantities in the text reflect the "standard error of the mean" for the five model structures. Molecular dynamics simulations were performed for a duration of $55 \mathrm{~ns}$ at 13 values of temperature (T), specifically [350, 400, $450,475,500,525,550,575,600,650,700,750,800] \mathrm{K}$, for each of the five replicas. In a video included in SI, we compare the trajectories of a

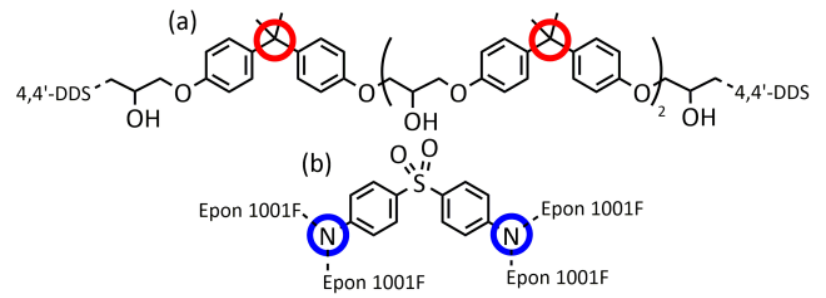

Figure 1. Chemical Structure of (a) Epon $1001 \mathrm{~F}$ and (b) 4,4'-DDS units in network. Two sets of atoms of interest: (i) central C and (ii) cross-link $\mathrm{N}$ atoms are highlighted by red and blue colored circles, respectively. 


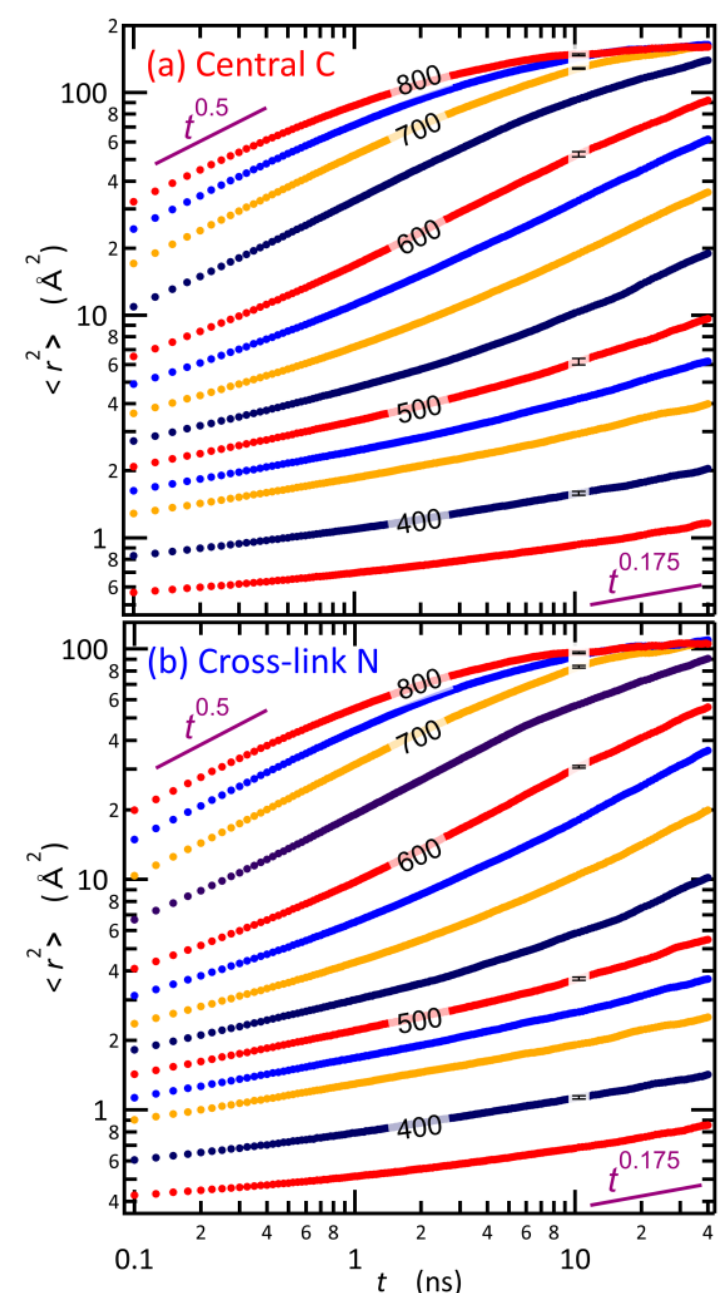

Figure 2. MSD ( $<r^{2}>$ ) vs. $t$ of (a) central C and (b) cross-link $\mathrm{N}$ atoms. Value of $T_{\mathrm{g}}$ is $489 \pm 1 \mathrm{~K}$. Representative error bars at labeled temperatures shown.

small part of the cross-linked network over a time span of 40 ns at temperatures both below and above $T_{\mathrm{g}}$ (400 $\mathrm{K}$ and $650 \mathrm{~K}$, respectively). In the same video, we also overlay the trajectories to visualize the conformational space explored by the network at the two temperatures.

We focus our analysis on two sets of atoms: (a) the central carbon atoms of the Bisphenol A moieties in the Epon 1001F units; and (b) the nitrogen atoms of the 4,4'-DDS units in the cross-linked network (Figure 1). These sets of atoms, which will be referred to as "central C" atoms and "cross-link N" atoms, respectively, are expected to show the fastest and the slowest dynamics in the cross-linked network because of their differing topological constraints. ${ }^{5}$ The log-log MSD trends vs. time for central $\mathrm{C}$ and cross-link $\mathrm{N}$ atoms over a duration of $40 \mathrm{~ns}$ are shown in Figure 2. Other simulation and analysis details are discussed in SI. As can be seen in the figures, both sets of atoms show similar qualitative behavior, although, as expected, the central $\mathrm{C}$ atoms are more mobile than the cross-link $\mathrm{N}$ atoms by a factor of about 1.5. For both sets, three observations can be noted. (1) The four MSD trends at temperatures below $T_{\mathrm{g}}(489 \mathrm{~K})$ show only a marginal increase in time scaling from approximately $t^{0.1}$ to about $t^{0.175}$ dependence. (2) Above $T_{\mathrm{g}}$, the MSD values increase to levels typical of molecular length scales and the time scaling peaks at about $t^{0.5}$. (3) For temperatures above $700 \mathrm{~K}$ at long times, the MSD trends plateau, which can be attributed to topological localization because of the highly cross-linked nature of the matrix. The $t^{0.5}$ subdiffusive behavior has been previously reported in atomistic $^{5}$ as well as coarse-grained simulations..$^{21,22}$ The localization plateau, on the other hand, has only been previously seen in coarse-grained simulations. ${ }^{21,22}$
Despite differences in network chemistry, these findings are largely consistent with those reported by Lin and Khare. ${ }^{5}$ However, one point of difference is that we are able to access the plateau values arising from topological localization because the highest temperature relative to $T_{\mathrm{g}}$ for the MSD trends studied here is 1.65 compared to 1.25 in their work..$^{23}$ Further, since our models are larger by a factor of $25,{ }^{24}$ the MSD trends shown here have far lower uncertainty. Thus, the possibility of collapsing the trends in Figure 2 to form a master curve for each set of atoms via TTSP is visually evident. To do this, we use the following relationship, which assumes thermorheological simplicity:25, 26

$$
b_{\mathrm{T}}^{-1}<r^{2}\left(T_{\mathrm{o}}, \mathrm{t} / a_{\mathrm{T}}\right)>=\left\langle r^{2}(T, t)>\right.
$$

where $\left\langle r^{2}(T, t)>\right.$ is the MSD, $a_{\mathrm{T}}$ and $b_{\mathrm{T}}$ is the time-shift and verticalshift factors, respectively, and $T_{0}$ is the reference temperature for the master curve. The quantity $t / a_{\mathrm{T}}$ is commonly referred to as the reduced time. We followed the recommendations of Dealy and Plazek ${ }^{26}$ for the vertical-shift factors, which are discussed further in SI.

The vertically shifted MSD trends are progressively shifted along the time axis until all the trends collapse onto a master curve (see the video in SI). This empirical protocol is performed for both sets of atoms, and the $a_{\mathrm{T}}-T$ trend is determined. In Figure 3a, the individual component trends comprising the master curve for the cross-link $\mathrm{N}$ atoms have been vertically offset to visualize the quality of the superposition effort. The resulting master curves as a function of the reduced time for the two sets of atoms are shown in Figure 3b. In Figure 3c, the slopes of the two master curves are shown, which quantifies the exponents of the time-dependence of the master curves. Finally, in Figure 3d, for the purpose of comparison, we show the experimental creep compliance master curve using data obtained from the literature. ${ }^{13}$

As can be seen in Figure 3a-b, we observe excellent superposition of the MSD trends for the two sets of atoms. The central C atoms are consistently more mobile than the cross-link $\mathrm{N}$ atoms, as would be expected from their differing topological context. The central $C$ atom is present on the relatively flexible Epon $1001 \mathrm{~F}$ units, while the cross-link $\mathrm{N}$ atom is the site where the rigid cross-linker units are each pinned down by four Epon 1001F units (Figure 1). However, since all the component molecular units form a highly cross-linked network, the overall character of the dynamics for each component is tightly coupled, as can be concluded from the strong qualitative resemblance of the two master curves. For both master curves, we find that the trends in their timedependence (Figure 3c), suggest the existence of three distinct regimes based on points of inflection and maxima for the curves. We discuss below the correspondence of these three regimes to the glassy, transition, and the rubbery states, respectively.

The superposition of the MSD trends allows us to quantitatively compare the three regimes identified in the simulations with the behavior of the creep compliance seen in experiments reported in the literature. ${ }^{13}$ In Figures $\mathbf{3 b}-\mathbf{d}$, we display dashed lines where each of the two master curves exhibit a point of inflection ( $t^{0.175}$ dependence) and a maximum ( $t^{0.5}$ dependence). We have identified these as empirical criteria which indicate a transition in material behavior. The time-dependence trends (Figure 3c) show that the behavior of the two sites is similar, but becomes slightly out of phase outside of the glassy regime. This phase difference provides us with two bounds of time corresponding to the atoms showing the fastest (central $\mathrm{C}$ atoms) and the slowest (cross-link $\mathrm{N}$ atoms) dynamics and allows us to identify relatively gradual rather than sharp boundaries between the three regimes. These softer criteria are more consistent with glassy dynamics than the sharp criteria observed in first-order phase transitions. As can be seen in Figure 3c, the central $C$ atoms consistently fulfill these criteria prior to the cross-link $\mathrm{N}$ atoms due to their greater topological mobility, as discussed above.

In regime 1 (the glassy state), we find that the time-dependence of MSD increases gradually from $t^{0.1}$ to $t^{0.175}$ in the first four decades in time $\left(10^{-1} \mathrm{~s}\right.$ to $\left.10^{3} \mathrm{~s}\right)$. During this time span, the values of the MSD are less than about $2 \AA^{2}$, which is less than the square of both the atomic radii of carbon and nitrogen atoms. ${ }^{15,16} \mathrm{We}$ attribute these small values of MSD to the vibration of these atoms within their crowded cage, as can be expected in the glassy state. The trajectory and conformational space explored by the network at $400 \mathrm{~K}$ shown in the video (SI) is consistent with the expectation for dynamics in the glassy state. Further, 


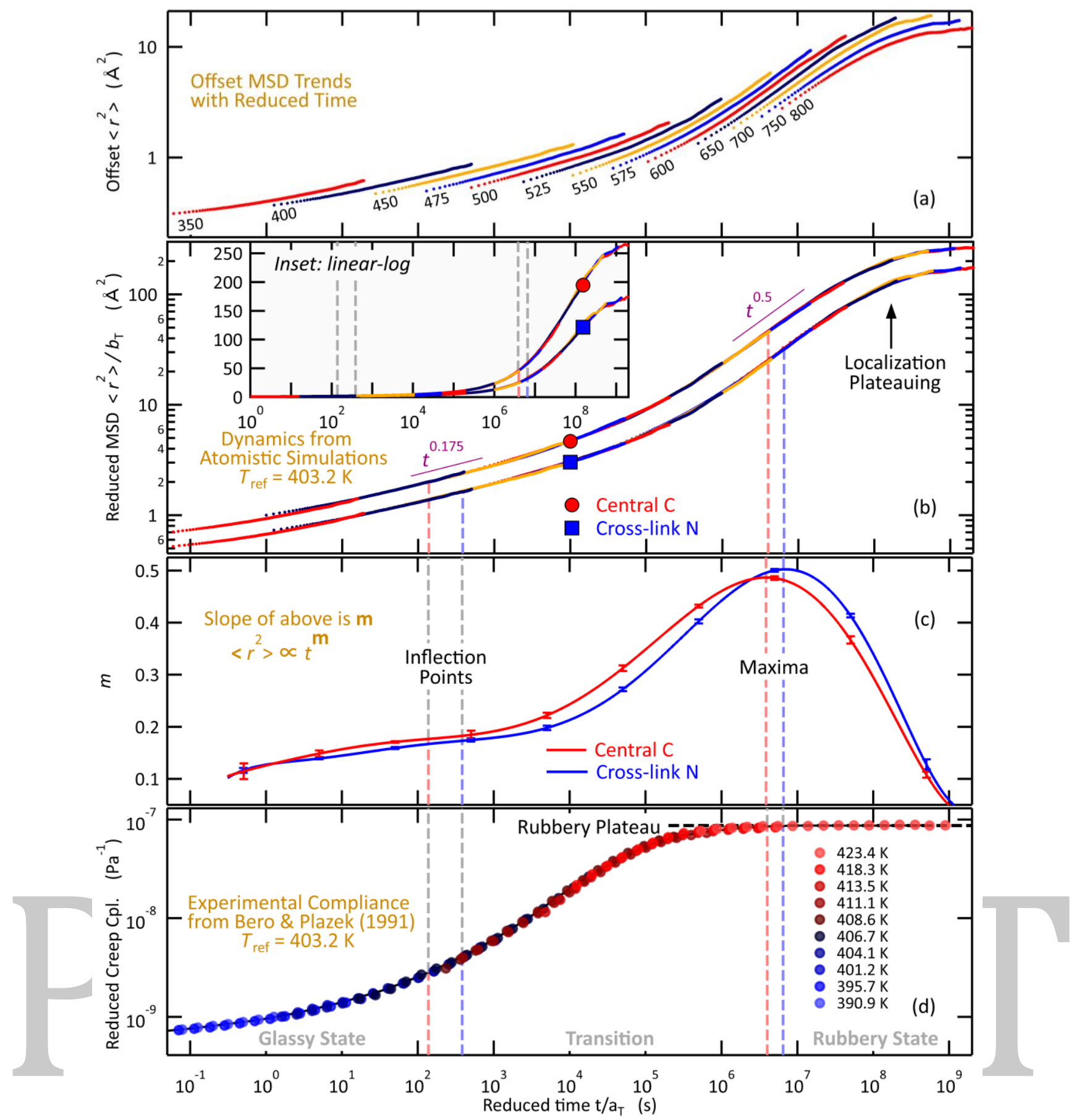

Figure 3. (a) Vertically Offset MSD trends with $t / a_{\mathrm{T}}$ from simulations at labelled temperatures to show quality of superposition. (b) Reduced MSDs from simulations. (c) Slopes of reduced MSDs. (d) Reduced creep compliance from experiments. Error bars are width of line for (a) and (b). Representative error bars shown for (c). Uncertainty estimates not available for $(\mathrm{d})$.

this attribution agrees with the trend in the creep compliance master curve that shows values typical of the glassy plateau during the same time span.

In regime 2, we find that the scaling of the reduced MSD increases sharply from approximately $t^{0.175}$ to $t^{0.5}$ dependence for both sets of atoms, and the value of the reduced MSD increases from about $2 \AA^{2}$ to about $40 \AA^{2}$ (Figure 3b). Corresponding to the abrupt increase in the MSD during this time span, the reduced creep compliance also exhibits a similar proportional increase from the glassy to the rubbery plateau as can be seen in Figure 3d. These observations are consistent with the fact that the data sets for this regime lie in the vicinity of the $T_{\mathrm{g}}$ for both simulation (Figure 3a-b) and experiment (Figure 3d). However, from Figure 3c, it is also found that in this regime, the time-dependence of the MSD trends for the two sets of atoms diverge, i.e., their slopes go

Official contribution of the U.S. National Institute of

Standards and Technology. Not subject to U.S. copyright. out of phase. Thus, unlike in the glassy state, the dynamics of the two sets of atoms are asynchronous in this regime.

The emergence of this asynchronous behavior in the network can be explained by the difference in the overall relative mobility for the two regimes. In the glassy state, all the atoms experience caging, and hence, the dynamics of the atoms in the network are tightly coupled with that of their neighbors. In the transition regime (which corresponds to either longer times or elevated temperatures), the atoms explore a far greater conformational space which varies based on their specific topological constraints (Figure 1). Thus, the atoms in the network exhibit topology-induced asynchronous (TIA) dynamics, which is seen as the divergence in Figure 3c. We note that the TIA dynamics phenomenon is distinct from the dynamic heterogeneity ${ }^{5,6,27,28}$ that is also implicated in the glass transition. The former is a temporal feature of the dynamics 


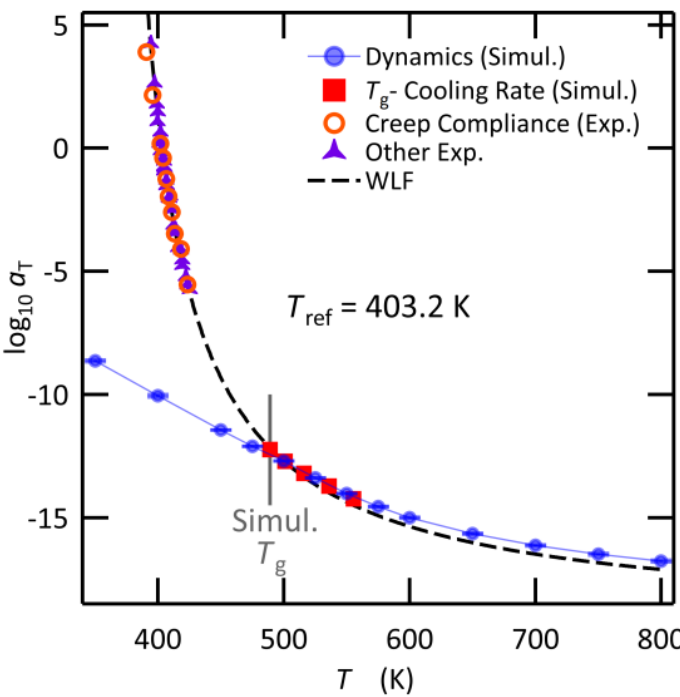

Figure 4. Time-shift factor $\left(a_{\mathrm{T}}\right)$ vs. $T$. Below simulation $T_{\mathrm{g}}, a_{\mathrm{T}}$ 's from simulations diverge from WLF as expected. Difference for two sets of atoms is captured in error bars.

of different groups of atoms, whereas the latter is a spatial feature of the dynamics of atoms. The TIA dynamics phenomenon is probably a characteristic of network polymers, where molecular units of disparate lengths and flexibilities are covalently bound to form a network.

The MSD master curves at the epoch of regime 3 ( $t^{0.5}$ dependence) were obtained at a temperature of $575 \mathrm{~K}$. This is the point where the specific volume trend studied in the previous work ${ }^{2}$ deviates from the rubbery line. In this regime, the values of the reduced MSDs show drastic increases as can be clearly seen in the inset of Figure 3b. Comparison with Figure 3d shows that macroscopically, this regime corresponds to the rubbery plateau in the experimental creep compliance trend. Comparison of Figures 3c and 3d indicates that the molecular origin of the onset of the rubbery plateau corresponds to the point where the maximum in diffusive behavior has been reached. Hence, we denote the time when the MSD master curves attain peak time-dependence ( $t^{0.5}$ dependence) as the epoch of rubbery behavior. At these elevated temperatures/long times, the molecular units of the network explore all available conformational space (see the video in SI). About a decade after the epoch of the rubbery behavior, the MSD trends begin to plateau due to topological localization. The peak and decline in the value of the slope in Figure 3c at the epoch of the rubbery regime indicate that, to some degree, localization starts at this point, but the effects are only slowly felt.

The designation of the $t^{0.175}$ and $t^{0.5}$ dependence (Figure 3c) as the bounds for the three regimes are based on empirical observations emergent from the simulation results. However, there is not any clear connection of those results to existing theory. Because of their underlying assumptions, neither the Rouse nor the Reptation model is rigorously applicable to cross-linked epoxy networks. ${ }^{29}$ However, $t^{0.25}$ dependence is predicted by de Gennes ${ }^{30}$ for Rouse-like diffusion within tube constraints, ${ }^{29}$ and $t^{0.5}$ dependence is predicted by primitive chain dynamics for entangled polymer melts. ${ }^{29}$ The latter dependence was also demonstrated using coarse-grained simulations, ${ }^{21,22}$ and theory ${ }^{31}$. While not exact, these results provide a frame of reference for interpreting the transition in the network dynamics that we observed here.

The empirically obtained $a_{\mathrm{T}}-T$ trend that is used to perform the superposition is shown in Figure 4. The values of $a_{\mathrm{T}}$ for the two sets of atoms are essentially identical, as would be expected from TTSP. This trend splices neatly with the trend that we previously calculated using specific volume-cooling rate analysis for the same network. ${ }^{2}$ Also in that figure, we show: (1) the experimental values of $a_{\mathrm{T}}$ extracted from the literature ${ }^{13}$; (2) the $a_{\mathrm{T}}$ values needed to superpose the experimental creep compliance trends extracted from the literature ${ }^{13}$ (Figure 3d); and (3) the $a_{\mathrm{T}}-T$ trend calculated using the material-specific William-Landel-Ferry (WLF) parameters in the literature. A gap exists between the time-shift factors obtained by experiment and simulations

Official contribution of the U.S. National Institute of

Standards and Technology. Not subject to U.S. copyright. due to the vast mismatch between the timescales accessed by the two methods. Nevertheless, as can be seen in the figure, this gap is neatly bridged by the WLF time-shift factor trend. Below the simulation $T_{\mathrm{g}}$ value of $489 \mathrm{~K}$, the time-shift factors obtained by superposing the simulation dynamics deviate from those calculated by the WLF relationship, as expected. These findings regarding the $a_{\mathrm{T}}-T$ trends are consistent with the previous work ${ }^{4}$ by one of us. Neither the experimental nor the WLF time-shift factors were used to guide our superposition effort here. The simulation $a_{\mathrm{T}}-T$ trend was obtained independently.

In the literature, ${ }^{32}$ a decoupling of segmental and chain dynamics in various polymers has been reported, which causes a breakdown in thermorheological simplicity and a failure of TTSP. We believe that due to the elevated temperatures used in our simulations and the highly cross-linked nature of the network, the two modes of dynamics remain coupled and our assumption of thermorheological simplicity appears to be valid. Other measures of translational and rotational dynamics of the network are currently being studied and will be included in a more detailed report.

To summarize, we studied the MSD trends of two sets of atoms in a cross-linked epoxy network using atomistic simulations. We find that these trends can be superposed using TTSP to form master curves. The temporal features of the reduced curves in simulations show excellent quantitative agreement with the creep compliance master curve obtained using experiments in the literature..$^{13}$ Finally, we find that the time-shift factors needed to obtain the master curves using simulation data show excellent agreement with those obtained from experimental literature data ${ }^{13}$ and our previously reported ${ }^{2}$ specific volume-cooling rate analysis. Altogether, such quantitative comparison between atomistic simulations and experiment presents an integrated view relating the molecular dynamics of the network with its macroscale viscoelastic characterization. Besides presenting a method to extend nanosecond simulations to macroscale timescales, these results should also be of interest to experimentalists. Many measurement techniques in current use infer structure and properties from the atomistic level dynamics, e.g., NMR and QENS. The results obtained here indicate that measurements of higher level segmental features could also be useful. Here, we show empirical evidence that such an approach is indeed productive. To the best of our knowledge, this is the first such report for network polymers.

\section{ASSOCIATED CONTENT}

\section{Supporting Information}

The Supporting Information is available free of charge.

1. Simulation details and vertical-shift factor (PDF)

2. Trajectory and conformation space of network at $400 \mathrm{~K}$ and $650 \mathrm{~K}$, and the progressive shifting to form master curve (MPG)

\section{AUTHOR INFORMATION}

\section{Corresponding Authors}

* (K.S.K.) E-mail ketan.khare@nist.gov.

* (F.R.P.Jr.) E-mail: frederick.phelan@nist.gov.

\section{ORCID}

Ketan S. Khare: 0000-0002-5487-5553

Frederick R. Phelan Jr.: 0000-0001-8004-5281

\section{Author Contributions}

K.S.K. and F.R.P.Jr. planned the study reported in this letter. K.S.K performed the simulations and analysis. Both authors were involved in writing the manuscript. A full-length research article that discusses the research presented in this letter is currently under review. Date of Preparation: June 26, 2019 


\section{Funding Sources}

U.S. National Institute of Standards and Technology grant number 70NANB16H005.

Notes

The authors declare no competing financial interest.

\section{ACKNOWLEDGMENT}

K.S.K is a Research Associate at the U.S. National Institute of Standards and Technology (NIST) and a Postdoctoral Fellow at Georgetown University. KSK gratefully acknowledges financial and facilities support by the Material Measurement Laboratory Professional Research Experience Program (PREP-MML) at NIST. This work used the Extreme Science and Engineering Discovery Environment (XSEDE), which is supported by the U.S. National Science Foundation Grant ACI-1053575. ${ }^{33}$ Certain commercial materials are identified in this paper to foster understanding. Such identification does not imply recommendation or endorsement by NIST, nor does it imply that the materials identified are necessarily the best available for the purpose.

\section{ABBREVIATIONS}

$T$, temperature; $T_{0}$, reference temperature; $T_{\mathrm{g}}$, glass transition temperature; $t$, time; MSD, mean squared displacement; TTSP, time-temperature superposition principle; 4,4'-DDS, 4,4'diaminodiphenyl sulfone; $a_{\mathrm{T}}$, time-shift factor; $b_{\mathrm{T}}$, vertical-shift factor, WLF, William-Landel-Ferry.

\section{REFERENCES}

(1) Materials Genome Initiative - Strategic Plan; Office of Science and Technology Policy: Washington, D.C. 20502, United States, 2014.

(2) Khare, K. S.; Phelan Jr., F. R. Quantitative Comparison of Atomistic Simulations with Experiment for a Cross-Linked Epoxy: A Specific Volume-Cooling Rate Analysis. Macromolecules 2018, 51, 564-575.

(3) Li, C.; Strachan, A. Molecular Scale Simulations on Thermoset Polymers: A Review. J. Polym. Sci., Part B: Polym. Phys. 2015, 53, 103122 .

(4) Sirk, T. W.; Khare, K. S.; Karim, M.; Lenhart, J. L.; Andzelm, J. W.; McKenna, G. B.; Khare, R. High Strain Rate Mechanical Properties of a Cross-linked Epoxy Across the Glass Transition. Polymer 2013, 54, 7048-7057.

(5) Lin, P.-H.; Khare, R. Local Chain Dynamics and Dynamic Heterogeneity in Cross-Linked Epoxy in the Vicinity of Glass Transition. Macromolecules 2010, 43, 6505-6510.

(6) Khare, K. S.; Khare, R. Effect of Carbon Nanotube Dispersion on Glass Transition in Cross-Linked Epoxy-Carbon Nanotube Nanocomposites: Role of Interfacial Interactions. J. Phys. Chem. B 2013, 117, 7444-7454.

(7) Khare, K. S.; Khabaz, F.; Khare, R. Effect of Carbon Nanotube Functionalization on Mechanical and Thermal Properties of CrossLinked Epoxy-Carbon Nanotube Nanocomposites: Role of Strengthening the Interfacial Interactions. ACS Appl. Mater. Interfaces 2014, 6, 6098-6110.

(8) Registered Trademark of Hexion Inc. Corporation, 180 East Broad St., Columbus OH 43215, United States.

(9) Epon $1001 \mathrm{~F}$ is a chemical trade name for the diglycidyl ether of bisphenol A (DGEBA) with the degree of polymerization of 2.

(10) Lemay, J. D.; Swetlin, B. J.; Kelley, F. N., Structure and Fracture of Highly Cross-linked Networks. In Characterization of Highly Crosslinked Polymers, American Chemical Society: 1984; Vol. 243, pp 165183.
(11) Choy, I.-C.; Plazek, D. J. The Physical Properties of Bisphenol-ABased Epoxy Resins During and After Curing. J. Polym. Sci., Part B: Polym. Phys. 1986, 24, 1303-1320.

(12) Plazek, D. J.; Choy, I. C. The Physical Properties of Bisphenol-ABased Epoxy Resins During and After Curing. II. Creep Behavior Above and Below the Glass Transition Temperature. J. Polym. Sci., Part B: Polym. Phys. 1989, 27, 307-324.

(13) Bero, C. A.; Plazek, D. J. Volume-Dependent Rate Processes in an Epoxy Resin. J. Polym. Sci., Part B: Polym. Phys. 1991, 29, 39-47.

(14) Plimpton, S. Fast Parallel Algorithms for Short-Range Molecular Dynamics. J. Comput. Phys. 1995, 117, 1-19.

(15) Wang, J.; Wang, W.; Kollman, P. A.; Case, D. A. Automatic Atom Type and Bond Type Perception in Molecular Mechanical Calculations. J. Mol. Graphics Modell. 2006, 25, 247-260.

(16) Wang, J.; Wolf, R. M.; Caldwell, J. W.; Kollman, P. A.; Case, D. A. Development and Testing of a General Amber Force Field. J. Comput. Chem. 2004, 25, 1157-1174

(17) General Amber Force Field (Version 1.8, March 2015) included in AmberTools 16.

(18) Dewar, M. J. S.; Zoebisch, E. G.; Healy, E. F.; Stewart, J. J. P. Development and Use of Quantum Mechanical Molecular Models. 76. AM1: A New General Purpose Quantum Mechanical Molecular Model. J. Am. Chem. Soc. 1985, 107, 3902-3909.

(19) Jakalian, A.; Bush, B. L.; Jack, D. B.; Bayly, C. I. Fast, Efficient Generation of High-quality Atomic Charges. AM1-BCC Model: I. Method. J. Comput. Chem. 2000, 21, 132-146.

(20) Jakalian, A.; Jack, D. B.; Bayly, C. I. Fast, Efficient Generation of High-quality Atomic Charges. AM1-BCC Model: II. Parameterization and Validation. J. Comput. Chem. 2002, 23, 1623-1641.

(21) Duering, E. R.; Kremer, K.; Grest, G. S. Structure and Relaxation of End-linked Polymer Networks. J. Chem. Phys. 1994, 101, 8169-8192.

(22) Kenkare, N. R.; Smith, S. W.; Hall, C. K.; Khan, S. A. Discontinuous Molecular Dynamics Studies of End-Linked Polymer Networks. Macromolecules 1998, 31, 5861-5879.

(23) Lin, P.-H.; Khare, R. Glass Transition and Structural Properties of Glycidyloxypropyl-Heptaphenyl Polyhedral Oligomeric Silsesquioxane-Epoxy Nanocomposites. J. Therm. Anal. Calorim. 2010, 102, 461-467.

(24) Lin, P.-H.; Khare, R. Molecular Simulation of Cross-Linked Epoxy and Epoxy-POSS Nanocomposite. Macromolecules 2009, 42, 43194327.

(25) Ferry, J., Viscoelastic Properties of Polymers. 3d ed.; Wiley: New York, 1980; p 641.

(26) Dealy, J.; Plazek, D. Time-Temperature Superposition: A Users Guide. Rheology Bulletin 2009, 78, 16-31.

(27) Long, D.; Lequeux, F. Heterogeneous Dynamics at the Glass Transition in van der Waals Liquids, in the Bulk and in Thin Films. Eur. Phys. J. E 2001, 4, 371-387.

(28) Lois, G.; Blawzdziewicz, J.; O'Hern, C. S. Percolation Model for Slow Dynamics in Glass-Forming Materials. Phys. Rev. E 2009, 102, 015702. (29) Doi, M.; Edwards, S. F., The Theory of Polymer Dynamics. Oxford University Press: United Kingdom, 1988; p 408.

(30) Gennes, P. G. d. Reptation of a Polymer Chain in the Presence of Fixed Obstacles. J. Chem. Phys. 1971, 55, 572-579.

(31) Vilgis, T. A.; Heinrich, G. Dynamics of Heterogeneous Polymer Networks. Phys. Rev. E 1994, 49, 2167-2174.

(32) Ding, Y.; Sokolov, A. P. Breakdown of Time-Temperature Superposition Principle and Universality of Chain Dynamics in Polymers. Macromolecules 2006, 39, 3322-3326.

(33) Towns, J.; Cockerill, T.; Dahan, M.; Foster, I.; Gaither, K.; Grimshaw, A.; Hazlewood, V.; Lathrop, S.; Lifka, D.; Peterson, G. D.; Roskies, R.; Scott, J. R.; Wilkins-Diehr, N. XSEDE: Accelerating Scientific Data. Comput. Sci. Eng. 2014, 16, 62-74. 


\section{Integration of Atomistic Simulation with Experiment using Time-Temperature Superposition for a Cross-linked Epoxy Network}

Ketan S. Khare*,t, and Frederick R. Phelan, Jr.*,

†Department of Physics, Georgetown University, Washington, DC 20057, United States

¥Material Science and Engineering Division, National Institute of Standards and Technology, Gaithersburg, MD 20899, United States

\section{Simulation Details}

All simulations were performed using the LAMMPS simulation package. ${ }^{1}$ The molecular models were described by the all-atom general AMBER force field (GAFF). ${ }^{2-4}$ The partial charges on atoms were calculated by the Austin Model 1 with bond charge correction model (AM1-BCC)..$^{5-7}$ The Van der Waals interactions were truncated at $9 \AA$, and the residual was accounted using tail corrections. ${ }^{8}$ The short-range pairwise Coulombic interactions were directly calculated up to $9 \AA$, and the long-range interactions were calculated using the particle-particle particle-mesh (pppm) method. ${ }^{9}$ Isothermal-isobaric conditions were maintained using the Nosé-Hoover ${ }^{10-12}$ thermostat and barostat. As described in the main manuscript, simulations were performed at 13 different values of temperature. The pressure, however, was maintained at $5 \mathrm{MPa}$ in each case. Further details about the preparation of the system are available in our previous work..$^{13}$ Simulations were started at snapshots saved at the end of the corresponding temperature step during the Slow-1× cooling rate in our previous work. ${ }^{13}$ Simulations were run for $55 \mathrm{~ns}$, and the coordinates for all heavy atoms were saved at intervals of $25 \mathrm{ps}$. We used the rerun command in LAMMPS ${ }^{1,14}$ to perform analysis.

\section{Vertical-Shift Factors $\left(b_{\mathrm{T}}\right)$}

Physically, the $a_{\mathrm{T}}-T$ trend quantitatively accounts for the drastic relationship between the polymer relaxation time and $T$, which causes the abruptness of the glass transition phenomenon. On the other hand, $b_{T}$ accounts for the relatively weak dependence of the stress magnitude on $T .{ }^{15}$ Since we are studying the MSD trends for the atoms in a highly cross-linked network, virial stress magnitudes will have a direct impact on the molecular friction associated with the translational dynamics in the rubbery state, effectively tending to slow down the dynamics, even though overall this tendency will be marginal compared to the direct effect of the temperature (i.e., acceleration) on the dynamics of the atoms in the network. ${ }^{15}$ This dependence of $b_{\mathrm{T}}$ on temperature is expected to cease below the $T_{\mathrm{g}}$ based on our previous experience. ${ }^{16}$ Therefore, we use the following relationship for the vertical shift factors:

$$
b_{\mathrm{T}}=\left\{\begin{array}{l}
\frac{\rho T}{\rho_{\mathrm{ref}} T_{\mathrm{ref}}} \text { if } T>T_{\mathrm{g}} \\
\frac{\rho_{\mathrm{T}} T_{\mathrm{g}}}{\rho_{\mathrm{ref}} T_{\mathrm{ref}}} \text { if } T<T_{\mathrm{g}}
\end{array},\right.
$$

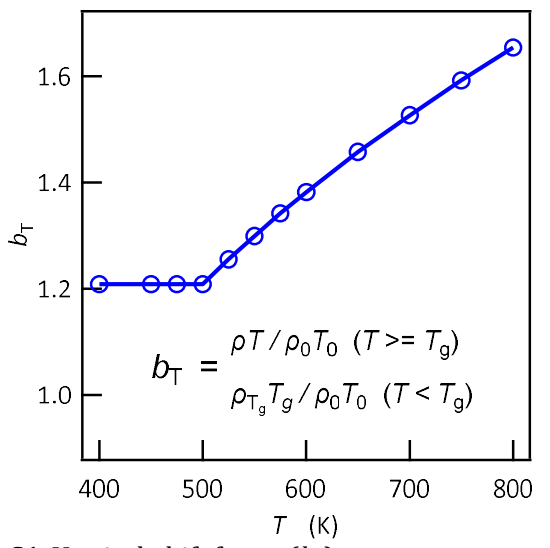

Figure S1. Vertical-shift factor $\left(b_{\mathrm{T}}\right)$ versus temperature $(T)$ where $\rho$, $\rho_{\text {ref }}$ and $\rho_{T_{\mathrm{g}}}$ are the densities at temperatures $T, T_{\text {ref, }}$ and $T_{\mathrm{g}}$ respectively. Since the simultaneous empirical fitting of both $a_{T}$ and $b_{T}$ causes both factors to lose physical significance, ${ }^{15}$ we have not made any attempts to improve our superposition effort by altering the values of $b_{\text {T }}$ from those calculated using equation $S 1$, which are shown in Figure S1. ${ }^{15}$ In any case, preliminary tests have shown that alterations to the values of $\mathrm{b}_{\mathrm{T}}$ have a negligible impact on the $a_{\mathrm{T}}-T$ trend of the network, which is of far greater relevance in this work.

\section{Trajectory and Conformational Space}

The trajectory of a small part of the network was visualized at $400 \mathrm{~K}$ and $650 \mathrm{~K}$ (below and above $T_{\mathrm{g}}$, respectively) using the Visual Molecular Dynamics ${ }^{17}$ (VMD) software. The trajectory was also overlaid to visualize the conformational space explored by the network (Figure S2). Both are shown in the video also included in the Supporting Information.

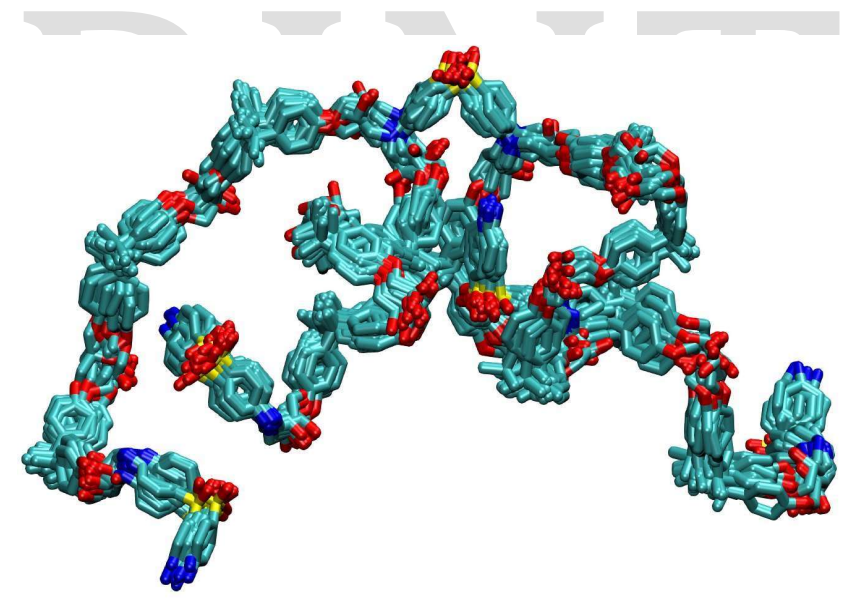

(a)

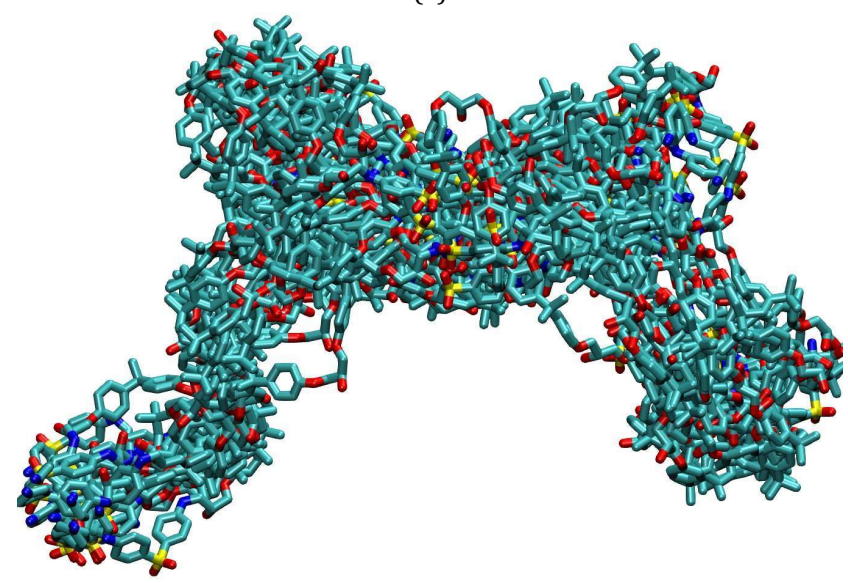

(b)

Figure S2. Trajectory of a small part of the network overlaid to show conformational space at (a) $400 \mathrm{~K}$ and (b) $650 \mathrm{~K}$ 


\section{Supporting Information}

\section{References}

(1) Plimpton, S. Fast Parallel Algorithms for Short-Range Molecular Dynamics. J. Comput. Phys. 1995, 117, 1-19.

(2) Wang, J.; Wang, W.; Kollman, P. A.; Case, D. A. Automatic Atom Type and Bond Type Perception in Molecular Mechanical Calculations. J. Mol. Graphics Modell. 2006, 25, 247-260.

(3) Wang, J.; Wolf, R. M.; Caldwell, J. W.; Kollman, P. A.; Case, D. A. Development and Testing of a General Amber Force Field. J. Comput. Chem. 2004, 25, 1157-1174.

(4) General Amber Force Field (Version 1.8, March 2015) included in AmberTools 16.

(5) Jakalian, A.; Bush, B. L.; Jack, D. B.; Bayly, C. I. Fast, Efficient Generation of High-quality Atomic Charges. AM1-BCC Model: I. Method. J. Comput. Chem. 2000, 21, 132-146.

(6) Jakalian, A.; Jack, D. B.; Bayly, C. I. Fast, Efficient Generation of High-Quality Atomic Charges. AM1-BCC Model: II. Parameterization and Validation. J. Comput. Chem. 2002, 23, 1623-1641.

(7) Dewar, M. J. S.; Zoebisch, E. G.; Healy, E. F.; Stewart, J. J. P. Development and Use of Quantum Mechanical Molecular Models. 76 AM1: A New General Purpose Quantum Mechanical Molecular Model. J. Am. Chem. Soc. 1985, 107, 3902-3909.

(8) Sun, H. COMPASS: An Ab Initio Force-Field Optimized for Condensed-Phase Applications Overview with Details on Alkane and Benzene Compounds. J. Phys. Chem. B 1998, 102, 7338-7364.

(9) Hockney, R. W.; Eastwood, J. W., Computer Simulation Using Particles. Institute of Physics Publishing: Philadelphia, 1988; p 267301.

(10) Nosé, S. A Unified Formulation of the Constant Temperature Molecular Dynamics Methods. J. Chem. Phys. 1984, 81, 511-519.

(11) Hoover, W. G. Canonical Dynamics: Equilibrium Phase-space Distributions. Phys. Rev. A 1985, 31, 1695-1697.

(12) Shinoda, W.; Shiga, M.; Mikami, M. Rapid Estimation of Elastic Constants by Molecular Dynamics Simulation Under Constant Stress. Phys. Rev. B 2004, 69, 134103.

(13) Khare, K. S.; Phelan Jr., F. R. Quantitative Comparison of Atomistic Simulations with Experiment for a Cross-Linked Epoxy: A Specific Volume-Cooling Rate Analysis. Macromolecules 2018, 51, 564-575.

(14) LAMMPS Manual, Sandia National Laboratory, Rerun command, URL: https://lammps.sandia.gov/doc/rerun.html.

(15) Dealy, J.; Plazek, D. Time-Temperature Superposition: A Users Guide. Rheology Bulletin 2009, 78, 16-31.

(16) Sirk, T. W.; Khare, K. S.; Karim, M.; Lenhart, J. L.; Andzelm, J. W.; McKenna, G. B.; Khare, R. High Strain Rate Mechanical Properties of a Cross-linked Epoxy Across the Glass Transition. Polymer 2013, 54, 7048-7057.

(17) Humphrey, W.; Dalke, A.; Schulten, K. VMD: Visual Molecular Dynamics. J. Mol. Graphics 1996, 14, 33-38.

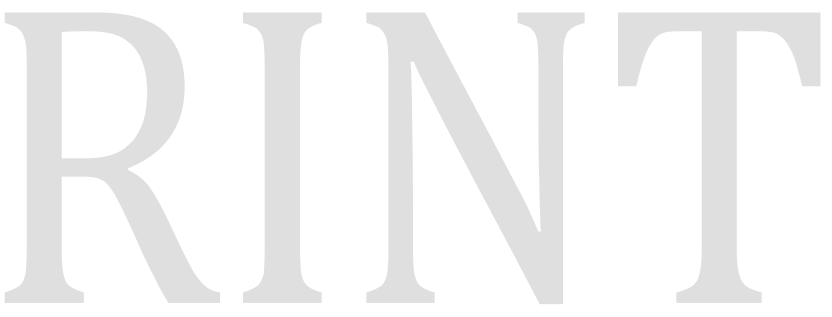


Other files 